\title{
Hybrid endovascular stent grafting for aortic arch aneurysm with aortopulmonary fistula
}

\author{
Kazuhiro Kochi, MD, Kenji Okada, MD, Masanobu Watari, MD, Kazumasa Orihashi, MD, and Taijiro Sueda, MD, \\ Hiroshima, Japan
}

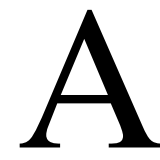

ortic arch replacement for aortic arch aneurysm with aortopulmonary fistula is not rare, but this procedure is still considered a challenge in aortic surgery. In one such case we used hybrid endovascular grafting without any dissection between the aneurysm and the lung.

\section{Clinical Summary}

A 72-year-old woman with aortic arch aneurysm was transferred to our unit for emergency surgery. The patient was medicated for severe chronic obstructive pulmonary disease. She presented with hoarseness and bronchial bleeding. A computed tomographic scan revealed an arch aneurysm measuring $7.5 \mathrm{~cm}$ in maximal diameter. Furthermore, the lung and pulmonary artery deviated inferiorly and anteriorly because of the aneurysm with thrombus. Impending rupture was suspected because of the pooling of pericardial effusion. Severely calcified plaque was also recognized in the ascending aorta (Figure 1). The location of the aortopulmonary fistula could not be identified.

A hybrid procedure of endovascular stent grafting and common arch replacement was proposed to avoid postoperative lung complications. The right subclavian artery was exposed and a polytetrafluoroethylene prosthetic graft $8 \mathrm{~mm}$ in diameter was attached. After median sternotomy, the pericardium was opened. The effusion consisted of old hematoma and bloody fluid. The aneurysm started at the anterior part of the aortic arch at the level of the left carotid

From the First Department of Surgery, Hiroshima University, School of Medicine, Hiroshima, Japan.

Received for publication June 6, 2001; accepted for publication July 24, 2001.

Address for reprints: Kazuhiro Kochi, MD, 1-2-3 Kasumi, Minami-ku, Hiroshima, 734-8551, Japan (E-mail: userdrkk@aol.com).

J Thorac Cardiovasc Surg 2002;123:363-4

Copyright (C) 2002 by The American Association for Thoracic Surgery

$0022-5223 / 2002 \$ 35.00+0 \quad \mathbf{1 2 / 5 4 / 1 1 9 0 6 1}$

doi:10.1067/mtc.2002.119061

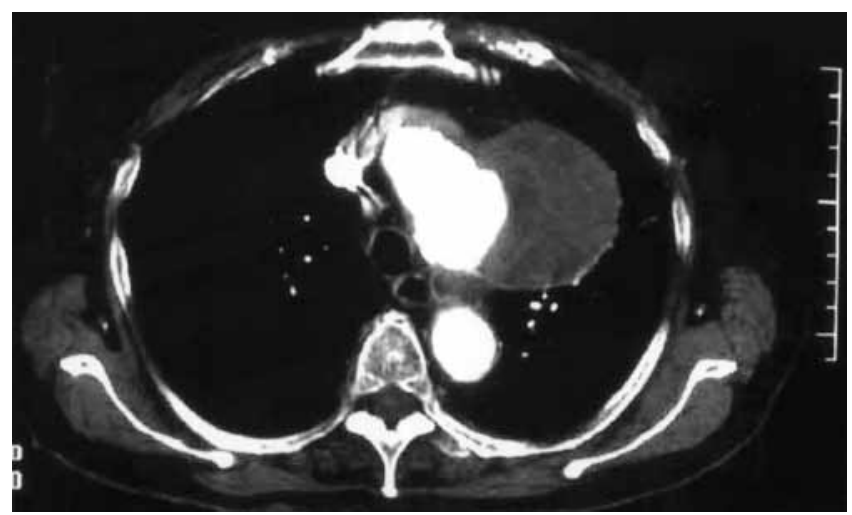

Figure 1. Preoperative computed tomographic scan demonstrating a huge aortic arch aneurysm involving arch vessels.

artery, protruded anteroinferiorly, and compressed the left pulmonary artery and right ventricular outflow tract. Cardiopulmonary bypass was established using the right atrium and the right subclavian artery through the conduit. Cervical vessels were exposed. Circulatory arrest was obtained at a rectal temperature of $25^{\circ} \mathrm{C}$. The anterior part of the arch was circumferentially transected. Cervical vessels were clamped. Selective cerebral perfusion was started through the conduit to the right brachiocephalic artery, directly through the left carotid artery and through the orifice of the left subclavian artery. The stent graft was made of a Gianturco stainless steel $\mathrm{Z}$ stent (Cook, Inc, Bloomington, Ind) inserted into a thin-walled Dacron graft with a diameter of $26 \mathrm{~mm}$ (Intervascular Medicals, Intervascular Inc, Clearwater, Fla). This stent graft was introduced through the $30 \mathrm{~F}$ sheath, deployed with the guidance of transesophageal echocardiography. The location and opening of the stent were ensured by dilation with a $20 \mathrm{~F}$ balloon. The proximal site of the graft was sutured circumferentially to the aortic wall. Distal and proximal anastomosis of the prosthetic graft was accomplished with a branched tube graft, and systemic perfusion 

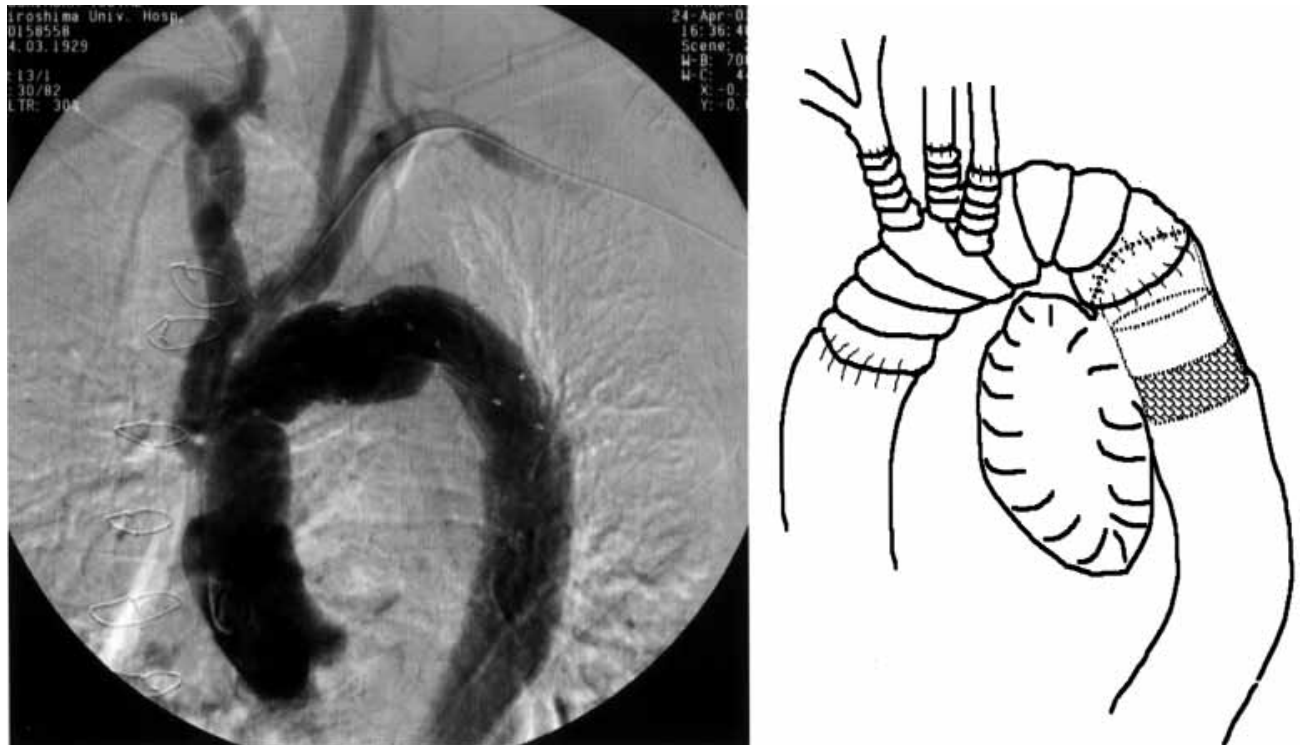

Figure 2. Postoperative angiogram and its schema.

was restarted through the fourth branch of the graft. Selective cerebral perfusion was stopped after the cervical vessels were reconstructed individually during the warming period. Weaning from bypass was achieved without difficulty. The patient was extubated on the next day and her postoperative course was uneventful. Postoperative angiography did not show any endovascular leakage at the stent graft (Figure 2). The patient was well 3 months after the operation, and repeated computed tomographic scans did not show any sign of endovascular leakage.

\section{Comment}

$\mathrm{We}^{1-3}$ previously reported the efficacy of endovascular stent grafting for distal aortic arch aneurysms occurring in patients undergoing another cardiac procedure and contraindicated for stent grafting through the femoral artery. The limitation of this method is difficulty in circumferential proximal anastomosis to the native aorta if the cervical vessels are involved in the aneurysm. ${ }^{4,5}$ Endovascular stent grafting with reconstruction of the cervical vessels for arch aneurysm was reported previously. ${ }^{4}$ However, that method might increase the risk of stroke because side clamping of the possibly diseased ascending aorta was required for the proximal anastomosis of the cervical grafts. Our strategy for endovascular stent grafting with reconstruction of the cervical vessels is as follows: (1) If the ascending aorta or thoracoabdominal aorta is severely diseased and the femoral arterial perfusion may cause retrograde thromboembolism, the right subclavian artery is selected as an arterial line for antegrade systemic perfusion ${ }^{3}$; (2) if the left subclavian artery needs to be reconstructed, the conduit can be used for reconstruction after decannulation of the arterial line through the conduit; (3) if other cervical vessels need to be reconstructed, common total arch replacement is performed after endovascular stent grafting (hybrid surgery).

The major advantage of the hybrid procedure is to avoid lung complications resulting from unnecessary dissection between the aneurysm and the lung. More experience is needed to confirm the surgical safety and long-term prognosis. However, this technique may increase survival in patients in whom surgery is contraindicated because of poor lung function.

\section{References}

1. Sueda T, Watari M, Orihashi K, Shikata H, Matsuura Y. Endovascular stent-grafting via the aortic arch for chronic aortic dissection combined with coronary artery bypass grafting. J Thorac Cardiovasc Surg. 1999;117:825-7.

2. Kochi K, Sueda T, Shibamura H, Orihashi K, Matsuura Y. Stent grafting to descending thoracic aorta during coronary artery bypass grafting. Ann Thorac Surg. 1999;68:1418-20.

3. Okada K, Sueda T, Orihashi K, Watari M, Ishii O. An alternative procedure of endovascular stent-graft repair for distal arch aortic aneurysm involving arch vessels. J Thorac Cardiovasc Surg. 2001; 121:182-4.

4. Kato M, Kaneko M, Kuratani T, Horiguchi K, Ikushima H, Ohnishi K. New operative method for distal aortic arch aneurysm: combined cervical branch bypass and endovascular stent-graft implantation. $J$ Thorac Cardiovasc Surg. 1999;117:832-4.

5. Mitchell RS, Dake MD, Semba CP, Fogarty TJ, Zarins CK, Liddell RP, et al. Endovascular stent-graft repair of thoracic aortic aneurysms. $J$ Thorac Cardiovasc Surg. 1996;111:1054-62. 